\title{
Model and optimal Call Admission Policy in Cellular Mobile Networks
}

\author{
Amine Berqia ${ }^{1}$ and Noufissa Mikou ${ }^{1}$ \\ ${ }^{1}$ Université de Bourgogne \\ UFR Sciences et Techniques \\ LIRSIA \\ BP 47870 \\ 21078 Dijon Cedex, France \\ \{berqia,mikou\}@crid.u-bourgogne.fr
}

\begin{abstract}
For current cellular networks, two important Quality of Service $(Q \circ S)$ measures are the fractions of new and handoff calls that are blocked due to unavailability of channels. Based on these QoS measures, we propose a queuing network model with impatient users for handoff and new calls in cellular mobile networks. The number of simultaneous calls, that can be supported, is modeled by $C$ identical servers with exponentially distributed session duration for each one of them. Priority is given to handoffs over new calls. We use for that a Guard Channel policy that reserves a set of $C_{H}$ channels for handoff calls, new calls being served at their arrival if there are more than $C_{H}$ available channels. We derive loss probabilities, mean waiting times in the queues, and we show that the Guard Channel policy is optimal for minimizing a linear objective function of the new and handoff calls. Simulation results illustrating the behavior of our system are given.
\end{abstract}

\section{Introduction}

During the last few years there is a great demand for PCS (Personal Communication Services) which will provide reliable voice and data communications anytime and anywhere. The service area in a PCS network is partitioned into cells. In each cell, a Base Station (BS) is allocated a certain number of channels which is assigned to the mobile terminal MT enabling the MT to communicate with other MT's. As the MT moves from one cell to another, any active call needs to be allocated a channel in the destination cell. This event is called a handoff. Allocating radio ressources to users with minimum blocking of new calls and dropping of handoff calls has become a vital issue in a mobile communication system design. It is known that dynamic channel allocation schemes can reduce these blocking and dropping probabilities for a reasonable range of loadings [2, 9]. However, allocating channels to every user whenever the sources are available may not be the optimal strategy in term of system performance.

The cellular network PCS have to be designed to guarantee the required Quality of Service $(Q \circ S)$ to each service or type of call (call meaning voice or data). Several policies and analysis are proposed in [4, 6] to minimize blocking 
of handoff calls. In our paper we investigate a call admission policy which gives priority to handoff attempts over new call attempts.

In this paper, we assume that the cellular network is homogeneous and thus, we can examine a single network cell in isolation. The radio ports are modeled by $C$ identical servers with exponentially distributed session duration for each one of them. We use a Guard Channel policy that reserves a set of $C_{H}$ channels for handoff calls, new calls being served at their arrival if there are more than $C_{H}$ available channels. Cellular networks performance was analysed in [6] using an approach where new and handoff calls were assumed to be Poisson arrival Processes. This approach is acceptable for macro cellular networks where a call might request for handoff only a few times. In this paper, we propose a Markov Modulated Poisson Process (MMPP) to describe voice handoffs (VH) arrival process because the arrival rate may depend on the state of the environment. The data Handoffs (DH) (as files transfer) and new calls (NC) arrive according to two different Poisson processes. Two separate finite buffer size queues are considered, one for each type of calls (handoff or new). The handoff calls enter service if there are available channels or join its queue if no idle channels are available and the queue is not full. Otherwise, They are lost. An arriving new call first checks the servers. It enters service if the number of busy servers is less than $C-C_{H}$, otherwise it enters the queue if the buffer is not full. Both handoff and new calls are impatient. That is a handoff leaves its queue and will be lost if it fails to get a channel before a Time Out (TO). And, in the same way, if a new user cannot get a channel before the end of the Patience Waiting Time (PWT), it leaves the queue. We derive loss probabilities, mean waiting times in both queues and we show that the Guard Channel policy is optimal for minimizing a linear objective function of the new and handoff calls. This paper is organized as follows: the queueing model is precisely defined in section 2. Loss probabilities and mean waiting time in queues are derived in section 3. In section 4, we show that the Guard Channel policy is optimal for minimizing a linear objective function of the new and handoff calls. Simulation results illustrating the behavior of our system are given and discussed in section 4. Finally, we conclude with some remarks in section 5 .

\section{The model}

We consider a system with two separate queues for handoff and new calls with priority given to handoff over new attempts.

It is assumed that voice handoffs arrive according to an MMPP process $A^{V H}$ with intensity $\lambda(\mathrm{t})=\sum_{\mathrm{i}=1}^{\mathrm{n}} \lambda_{\mathrm{i}} \times 1_{[\mathrm{Y}(\mathrm{t})=\mathrm{i}]}$ at time $t$ where:

- $(Y(t))$ is an irreducible Markov chain with state space $\{1,2, \ldots, \mathrm{n}\}$ and an infinitesimal generator denoted by $Q_{n}$; 
$-\lambda_{i}, i=1 . . n$, are non-negative numbers;

and

$$
-1_{[Y(t)=i]}=\left\{\begin{array}{l}
1 \text { if } Y(t)=i \\
0 \text { otherwise. }
\end{array}\right.
$$

We suppose that the process of data handoff attempts is a Poisson process denoted by $A^{D H}$ with arrival rate $\lambda_{D H}$ and that mobile new users generate calls with a Poisson arrival denoted by $A^{N C}$ with intensity $\lambda_{N C}$.

The service is provided by $C$ channels with exponentially distributed session duration for each one of them. We use a Guard Channel policy that reserves a set of $C_{H}$ channels for handoff calls, new calls being served at their arrival if there are more than $C_{H}$ available channels. The evolution of the system for both new

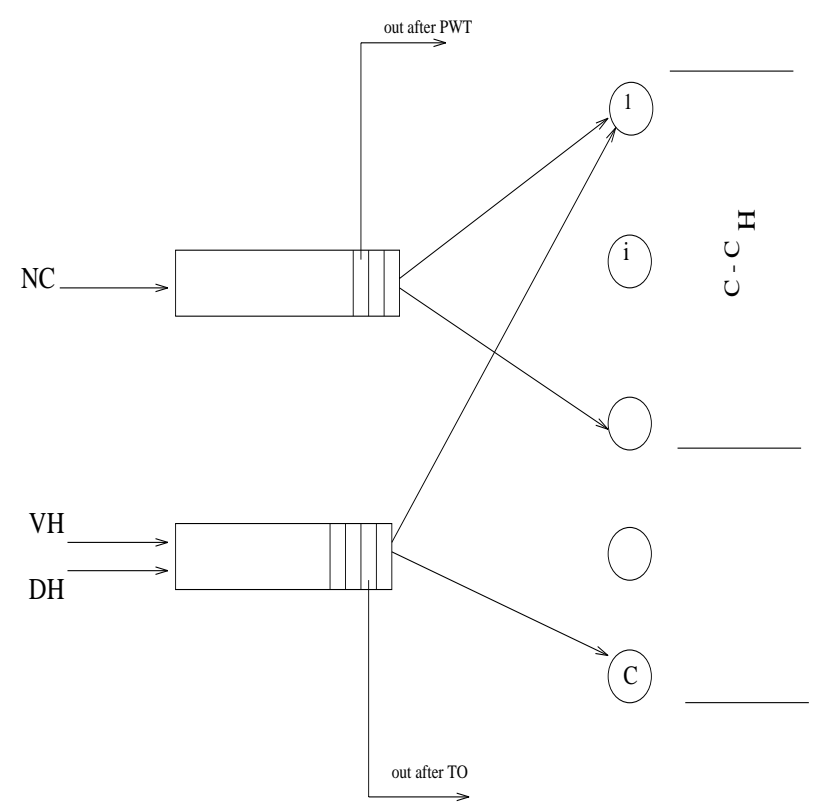

Fig. 1. The Model

and handoff traffic flow is as follows:

- An arriving handoff call first checks the servers. It enters service if one of them is available. Otherwise, it is queued if the buffer is not full. A handoff in the queue will be lost if it fails to get a channel before a Time Out (TO).

- An arriving new call first checks the servers. It enters service if the number of busy servers is less than $C-C_{H}$, otherwise it enters its queue if the buffer is not full. If a new user cannot get a channel before the end of the Patience Waiting 
Time (PWT) it will be lost.

The Patience Waiting Time (PWT) and the Time Out (TO) are distributed exponentially with mean $1 / \mu_{N}$ and $1 / \mu_{H}$ respectively.

The queue buffer sizes are finite and denoted by $K_{H}$ for handoff traffic and $K_{N}$ for new calls.

Therefore, the new call generated by the mobile user calls may be lost at two cases: if the coresponding buffer is full at its arriving time or if its waiting time is greater than PWT. A handoff call may be lost if the corresponding buffer is full or if it fails to get a channel before that the TO expires.

The system is described by the multidimensional Markov Chain :

$$
\left(X_{V H}(t), X_{D H}(t), X_{N C}(t), S(t), Y(t)\right)
$$

where:

- $X_{V H}(t)$ (respectively $X_{D H}(t)$ ) is the number of voice Handoffs (respectively data handoffs) in their queue at time $t$ respectively,

- $X_{N C}(t)$ is the number of new calls at time $t$ in their queue,

- $S(t)$ is the number of busy servers at time $t$,

and

- $Y(t)$ is the phase of the MMPP representing the state of the environment.

In this paper, we analyse our system in the steady state.

\section{Performance analysis}

Let $B=\left\{(i, j, k, l, m) / 0 \leq i+j \leq K_{H}, 0 \leq k \leq K_{N}, 0 \leq l \leq C, 1 \leq\right.$ $m \leq n\}$ be the state space of the precedent multidimensional Markov Chain and $P(i, j, k, l, m)$ be the stationary probability that the system is in the state $(i, j, k, m, n)$.

The $P(i, j, k, l, m)((i, j, k, l, m) \in B)$ verify the normalization condition :

$$
\sum_{(i, j, k, l, m) \in B} P(i, j, k, l, m)=1
$$

A VH or DH call is lost if the corresponding buffer is full or if its waiting time in the queue exceeds a Time Out (TO).

The probability that a VH mobile user attempt finds the buffer full is given by :

$$
\tilde{P}_{V H}=\frac{\sum_{i+j=K_{H}} \sum_{k=0}^{K_{N}} \sum_{m=1}^{n} \lambda_{m} P(i, j, k, C, m)}{\sum_{m=1}^{n} \lambda_{m} \pi_{m}}
$$

where $\pi_{Y}=\left(\pi_{1}, \ldots, \pi_{n}\right)$ is the unique vector probability solution of $\pi_{Y} Q_{n}=0$ 
(where $Q_{n}$ is the infinitesimal generator of $\mathrm{Y}$ ).

A DH call finds the buffer full upon arrival with probability $\tilde{P}_{D H}$ given by :

$$
\tilde{P}_{D H}=\sum_{i+j=K_{H}} \sum_{k=0}^{K_{N}} \sum_{m=1}^{n} P(i, j, k, C, m)
$$

If the time out expires before that a handoff gets a channel, the call is lost. The loss probability due to time out is given by :

$$
P_{V T O}=\frac{\sum_{(i, j, k, l, m) \in B / i>0} i \mu_{H} P(i, j, k, l, m)}{\left(\sum_{m=1}^{n} \lambda_{m} \pi_{m}\right)\left(1-\tilde{P}_{V H}\right)}
$$

for the voice handoffs

$$
P_{D T O}=\frac{\sum_{(i, j, k, l, m) \in B / j>0} j \mu_{H} P(i, j, k, l, m)}{\lambda_{D H}\left(1-\tilde{P}_{D H}\right)}
$$

for the data handoffs

So, the total loss probability for voice and data handoffs is respectively :

$$
P_{V H}=\tilde{P}_{V H}+\left(1-\tilde{P}_{V H}\right) P_{V T O}
$$

for the voice handoffs

$$
P_{D H}=\tilde{P}_{D H}+\left(1-\tilde{P}_{D H}\right) P_{D T O}
$$

for the data handoffs

In the same way, a new call is lost if at its arrival the corresponding buffer is full or if its waiting time in the queue exceeds the Patience Waiting Time (PWT).

The probability that a NC finds the buffer full is given by :

$$
\tilde{P}_{N C}=\sum_{i+j=0}^{K_{H}} \sum_{m=1}^{n} \sum_{l=C_{H}}^{C} P\left(i, j, K_{N}, l, m\right)
$$

The loss probability $\tilde{P}_{N C}$ due to PWT is given by :

$$
\tilde{P}_{P W T}=\frac{\sum_{(i, j, k, l, m) \in B / k>0} k \mu_{N} P(i, j, k, l, m)}{\lambda_{N C}\left(1-\tilde{P}_{N C}\right)}
$$


So, the total loss probability for new calls is given by :

$$
P_{N C}=\tilde{P}_{N C}+\left(1-\tilde{P}_{N C}\right) P_{P W T}
$$

The mean number of VH in the queue is given by :

$$
E(V H)=\sum_{(i, j, k, l, m) \in B} i P(i, j, k, l, m)
$$

In the same way, we obtain the mean number of $\mathrm{DH}$ and $\mathrm{NC}$ in the queue :

$$
\begin{aligned}
& E(D H)=\sum_{(i, j, k, l, m) \in B} j P(i, j, k, l, m) \\
& E(N C)=\sum_{(i, j, k, l, m) \in B} k P(i, j, k, l, m)
\end{aligned}
$$

and

$$
E(D N)=\sum_{(i, j, k, l, m) \in B} l P(i, j, k, l, m)
$$

The mean waiting time in the queue $W$, for voice handoffs, data handoffs and new calls can be obtained using Little's formula [1].

So, we have :

$$
\begin{gathered}
W(V H)=\frac{E(V H)}{\left(\sum_{m=1}^{n} \lambda_{m} \pi_{m}\right)\left(1-\tilde{P}_{V H}\right)} \\
W(D H)=\frac{E(D H)}{\lambda_{D H}\left(1-\tilde{P}_{D H}\right)}
\end{gathered}
$$

and

$$
W(N C)=\frac{E(N C)}{\lambda_{N C}\left(1-\tilde{P}_{N C}\right)}
$$

The probabilities $P(i, j, k, l, m)$ are numerically obtained using DNAmaca [5] which provides performance analysis sequence including steady state solution. In section 4, we prove that the Guard Channel policy is an optimal policy. 


\section{Optimal Admission Policy}

In this section, we consider the problem of finding an optimal admission policy that determines the acceptance or rejection of new and handoff calls. For the cellular system described in section 3 , let $\mathrm{S}(\mathrm{t})$ be the Markov chain representing the number of busy channels at time $t$. If, at steady state, that Markov chain is in state $l$, the policy consists then to choose an action $a(l)$ in a set $A$ of acceptance/reject actions depending on the state $l$, as follows : a call will be rejected due to impatience time or if there is no available channel and the buffer is full, otherwise it will be accepted. When the system is in state $l$ and action $a$ is chosen, then we incur a cost $C(l, a)$ which will be $L_{h}$ or $L_{n}$ according to the action is a handoff or a new call reject. Moreover, the next state of the system is chosen according to the transition probabilities $P_{l j}(a)$.

Our problem is to find a policy $\pi^{*}$ over all call admission control policies $\pi$ such that :

$$
\phi_{\pi^{*}}=\min _{\pi} \phi_{\pi}
$$

where

$\phi_{\pi}=\lim _{N \rightarrow+\infty} E_{\pi}\left(\sum_{k=0}^{N-1} L_{h} \pi_{h k}+\sum_{k=0}^{N-1} L_{n} \pi_{n k}\right)$

$E_{\pi}$ represents the conditional expectation given that the policy $\pi$ is employed

and

the binary mapping $\pi_{h k}, \pi_{n k}$ describing the actions for each state of the sytem are defined by :

$$
\begin{aligned}
& \pi_{h k}=\left\{\begin{array}{l}
1 \text { if } k^{\prime} \text { th handoff is rejected } \\
0 \text { otherwise. }
\end{array}\right. \\
& \pi_{n k}=\left\{\begin{array}{l}
1 \text { if } k^{\prime} \text { th new call is rejected } \\
0 \text { otherwise. }
\end{array}\right.
\end{aligned}
$$

we need the following:

Lemma 1. If there exists a bounded function $h(l), l=0,1, \ldots$, and a constant $g$ such that:

$$
g+h(l)=\min _{a}\left\{C(l, a)+\sum_{j=0}^{\infty} P_{l j}(a) h(j)\right\} ; l \geq 0
$$

then there exists a stationary policy $\pi^{*}$ such that :

$$
g=\phi_{\pi^{*}}(l)=\min _{\pi} \phi_{\pi}(l)
$$


For the proof of lemma 2, see [8], page 144.

We obtain the following :

Theorem 2. an optimal policy for the problem (18) is a Guard Channel policy. Now, we prove the existence of a bounded function $h(l)$ and a constant $g$ using the following construction:

For a discount factor $\alpha \in(0,1)$ and any policy $\pi$, we define :

$$
V_{\pi}(l)=E_{\pi}\left[\sum_{n=0}^{\infty} \alpha^{n} C\left(l_{n}, a_{n}\right) \mid l_{0}=l\right], l \geq 0
$$

where $E_{\pi}$ represents the conditional expectation given that the policy $\pi$ is employed and the initial state is $l$.

Let,

$$
V_{\alpha}(l)=i n f_{\pi} V_{\pi}(l)
$$

A policy $\pi^{*}$ is said to be $\alpha$-optimal if :

$$
V_{\pi^{*}}(l)=V_{\alpha}(l) \forall l \geq 0
$$

For the proof of the theorem 2, we use the lemma 1.

We denote $V_{\alpha}^{k}(l)$ the optimal cost for the $k$-stage starting in state $l$.

The $\alpha$-optimal cost function $V_{\alpha}($.$) for 0 \leq l \leq C$ satisfies :

$$
\begin{aligned}
& V_{\alpha}^{k}(l)= \\
& \lambda_{h} \min \left(V_{\alpha}^{k-1}(l+1), L_{h}+V_{\alpha}^{k-1}(l)\right) \\
& +\lambda_{n} \min \left(V_{\alpha}^{k-1}(l+1), L_{n}+V_{\alpha}^{k-1}(l)\right) \\
& +l \mu V_{\alpha}^{k-1}(l-1)+(C-l) \mu V_{\alpha}^{k-1}(l)
\end{aligned}
$$

Where $\lambda_{h}=\lambda_{V H}+\lambda_{D H}$ and $\lambda_{n}=\lambda_{N C}$.

We consider that $V_{\alpha}^{k}(C+1)=\infty$ and $V_{\alpha}^{k}(-1)=V_{\alpha}^{k}(0)$.

We define:

$$
V_{\alpha}(l)=\lim _{N \rightarrow+\infty} V_{\alpha}^{k}(l)
$$

It is shown in [10] that this function is non-decreasing and convex. Moreover, since the state space $B$ of our system defined in section 3 is finite and the Markov chain induced by the control policy is irreductible, $V_{\alpha}(l)-V_{\alpha}(0)$ is bounded uniformly according to [8], page 146 .

Let us define :

$$
h_{\alpha}(l)=V_{\alpha}(l)-V_{\alpha}(0)
$$

to be the $\alpha$-cost of state $l$ relative to state 0 .

Then, from equations (24) and (25), we obtain : 


$$
\begin{aligned}
& (1-\alpha) V_{\alpha}(0)+h_{\alpha}(l)= \\
& \lambda_{h} \min \left(h_{\alpha}(l+1), L_{h}+h_{\alpha}(l)\right) \\
& +\lambda_{n} \min \left(h_{\alpha}(l+1), L_{n}+h_{\alpha}(l)\right) \\
& +l \mu h_{\alpha}(l-1)+(C-l) \mu h_{\alpha}(l)
\end{aligned}
$$

Now, for some sequence $\alpha_{n} \rightarrow 1$ :

$h_{\alpha_{n}}$ converges to a function $h(l)$ and $\left(1-\alpha_{n}\right) V_{\alpha_{n}}(0)$ conerges to a constant $g$, then we obtain that:

$$
\begin{aligned}
g+h(l)= & \lambda_{h} \min \left(h(l+1), L_{h}+h(l)\right) \\
& +\lambda_{n} \min \left(h(l+1), L_{n}+h(l)\right) \\
& +l \mu h(l-1)+(C-l) \mu h(l)
\end{aligned}
$$

Now, we are able to apply lemma 1 to say that a stationary policy $a(l)$ verifying

$$
a(l)=\phi_{\pi^{*}}(l)=\min _{\pi} \phi_{\pi}(l)
$$

exists, and to give a:

\section{Proof of Theorem 2}

The optimal policy $a(l)$ that satisfies $(26)$ is given by :

$$
a(l)=\left\{\begin{array}{l}
1 \text { if } h(l+1)-h(l) \leq L_{n} \\
0 \text { otherwise }
\end{array}\right.
$$

Since $h(l)$ is non-decreasing and convex, $h(l+1)-h(l)$ is non-decreasing in $l$. Hence, there exist integers $i_{0}$ and $i_{1}$ where:

$$
i_{0}=\inf \left\{l: h(l+1)-h(l)>L_{n}\right\}
$$

and

$$
i_{1}=\inf \left\{l: h(l+1)-h(l)>L_{h}\right\}
$$

we note that $i_{1}>i_{0}$ since $L_{h}>L_{n}$.

So, the Guard channel policy that reserves $C-i_{0}$ channels for handoff calls is the optimal policy for our problem. 


\section{$5 \quad$ Numerical and Simulation results}

In this section, we present numerical and simulation results. For all experiments we consider $C=18$ servers whith exponentially distributed session duration of parameter $\mu=1$.

Figures Fig.2 and Fig.3 represent loss probabilities versus Handoff and new calls buffer size respectively. In both cases, the parameters $C_{H}$, PWT and TO are respectively 5, 40 and 30 . When the buffer size increases, we note that the loss probability of both Handoff and new calls decreases, and the handoffs loss probability is lower. Figures Fig.4 and Fig.5 depict loss probabilities of handoff

Loss probability

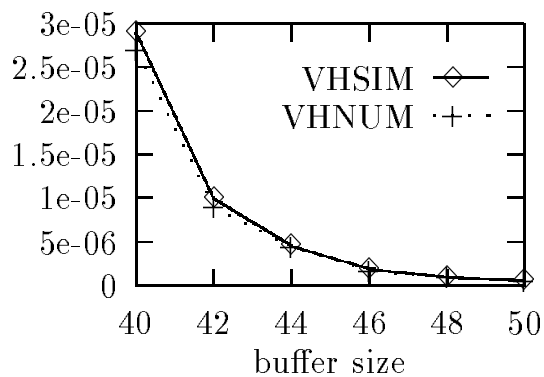

Loss probability

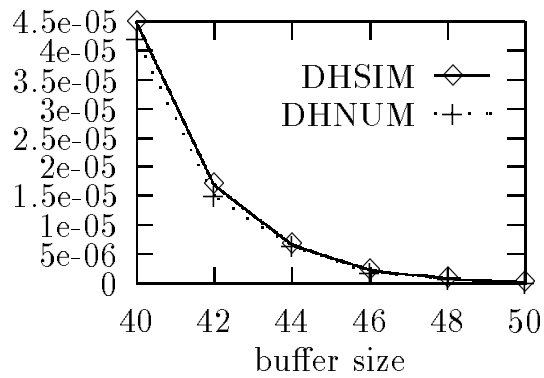

Fig. 2. handoffs loss probabilities vs. buffer size, $C=18, C_{H}=5, P W T=40, T O=30$

Loss probability

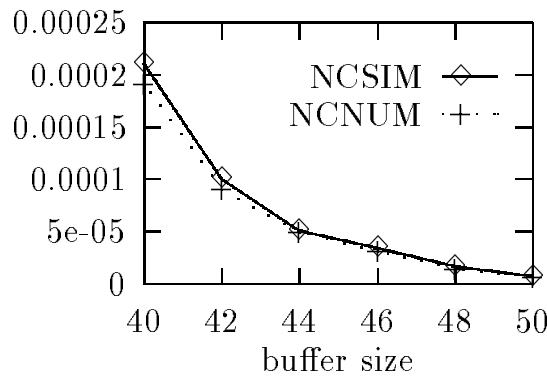

Fig. 3. new call loss probabilities vs. buffers size, $C=18, C_{H}=5, P W T=40, T O=30$

and new calls versus number of guard channels $C_{H}$. Loss probability decreases 
for Handoff mobile users when we increase the number of guard channels. The Loss probability of new calls increases when we increase the number of guard channels.

In Figures Fig. 4 and Fig.5 we see that if we reserve 3 or 4 channels from 18 channels, the Handoff loss probability is lower than $10^{-6}$, and the new call loss probability does not exceed the value $10^{-4}$. So, we propose to reserve $20 \%$ of channels to Handoffs.

The effect of channel reservation on the response times is depicted in Fig.6.

Loss probability

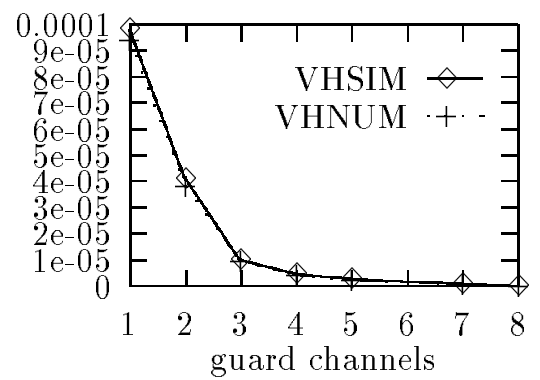

Loss probability

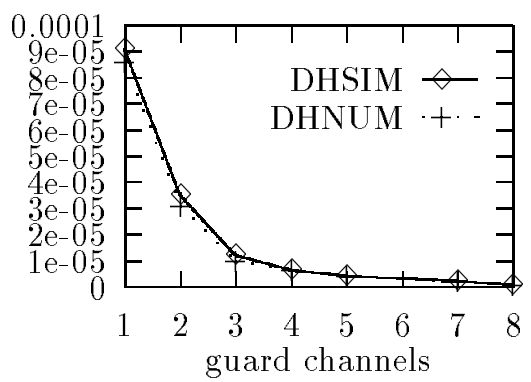

Fig. 4. handoffs loss probabilities vs.guard channels, $C=18, P W T=40, T O=30$

Loss probability

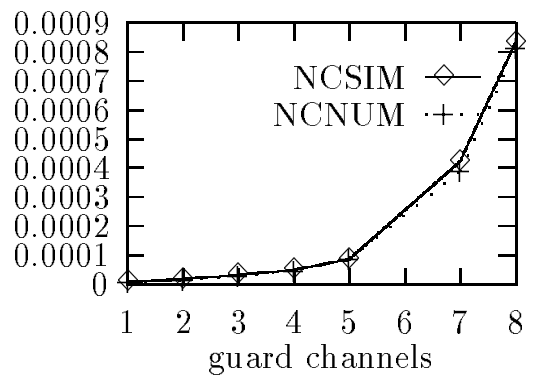

Fig. 5. new call loss probabilities vs.guard channels, $C=18, P W T=40, T O=30$ 
response time

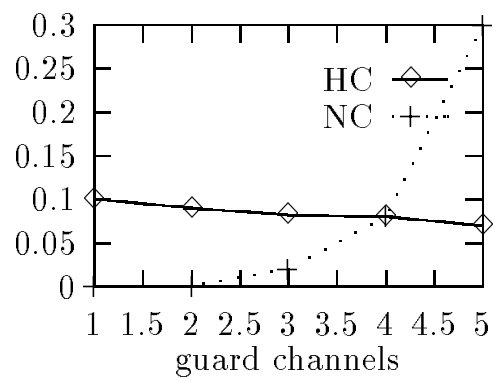

Fig. 6. response time vs. number of guard channels

\section{Conclusion}

In this paper, we have considered a Guard channel policy that gives priority to handoff over new calls in a cellular mobile network. We show that this policy is optimal for a linear objective function of the new and handoff call blocking probabilities. Under this policy, performance for handoffs are optimal without serious degradation for new calls. Moreover, blocking probabilities and response times for both types of calls are obtained and numerical results show the impact of the buffer size and the number of guard channels on the system behaviour. Finally Simulation results are conducted to validate our results.

\section{References}

1. F. Bacceli and P. Brémaud, Elements of Queueing Theory, Springer Verlag, Series Applications, August 1993.

2. R. Beck and H. Panzer, Strategies for handover and dynamic channel allocation in micro-cellular mobile radio systems, Vehicular Technology Conference, 1989.

3. D. P. Bertsekas, Dynamic Programming: Deterministic and Stochastic models, Prentice Hall, Englewood Cliffs, NJ, 1987.

4. R. Guérin, Queueing Blocking System with Two Arrival Streams and Guard Channels, IEEE Trans. on Communication, vol. 36, no.2, pp. 153-163, Feb. 1988.

5. W. J. Knottenbelt, DNAmaca: A Generalized Performance Analyser for Timed Transition Systems, Master's Thesis, University of Cape Town, South Africa, 1995.

6. D. W. Mc Millan, Delay Analysis of a Cellular Mobile Priority Queueing System, IEEE/ ACM Trans. on Networking vol. 3, no. 3, pp. 310-319, Jun. 1995.

7. I. Mitrani Prbabilistic Modeling, Cambridge University PRESS 1998.

8. S. Ross, Applied Probability Models with Optimization Applications, Holden-Day, 1970.

9. M. Zhang and T. S. P. Yum, Comparisons of channel assigment startegies in cellular mobile telephone systems, IEEE Transactions on Vehicular Technology, 38(4), November 1989.

10. Jean Walrand, An introduction to queueing networks, Prentice-Hall, Englewood Cliffs, NH, 1988. 